\title{
The contribution of silence to spiritual care at the end of life: a phenomenological exploration from the experience of palliative care chaplains
}

\author{
Lynn Bassett, Amanda F. Bingley \& Sarah G. Brearley
}

To cite this article: Lynn Bassett, Amanda F. Bingley \& Sarah G. Brearley (2018) The contribution of silence to spiritual care at the end of life: a phenomenological exploration from the experience of palliative care chaplains, Journal for the Study of Spirituality, 8:1, 34-48, DOI: 10.1080/20440243.2018.1431034

To link to this article: https://doi.org/10.1080/20440243.2018.1431034

\section{曲 Published online: 25 Feb 2018.}

\section{Submit your article to this journal $\sqsubset$}

Џll Article views: 38

Q View related articles ¿

View Crossmark data ¿ 


\title{
The contribution of silence to spiritual care at the end of life: a phenomenological exploration from the experience of palliative care chaplains
}

\author{
Lynn Bassett, Amanda F. Bingley and Sarah G. Brearley
}

Division of Health Research, Faculty of Health and Medicine, Lancaster University, Lancaster, UK

\begin{abstract}
At the end of life, silence often takes increasing prominence in caregiving encounters. Valued in spiritual and religious traditions, silence lends itself to the spiritual and existential dimensions of healthcare but lack of familiarity with the phenomenon can lead to anxiety or avoidance. Greater understanding of the contribution of silence to care may support professional caregiving practice. This paper reports research that explored the nature, meaning and value of silence in palliative spiritual care. In a two-phase phenomenological approach, data were gathered through self-inquiry and unstructured interviews with 15 palliative care chaplains. A descriptive and hermeneutic analysis facilitated explication of the lived experience to produce an interpretation of essential qualities of silence in this context. 'Spiritual caregiving silence' emerged as a person-centred phenomenon that supports patients and their relatives. It is described as a way of being with another person, complementary to speech and non-verbal communication, which evokes a sense of companionship and connection. The caregiver takes both active and participative roles in the silence to create an accompanied space that allows the other person in the relationship to be with her or himself in a way that may not be possible when alone. This demands engagement and commitment. Silence provides a means of, and a medium for, communication that is beyond the capacity of words and has the potential to enable change. This insight into the specialist experience of chaplains may resonate with the experience of other professional caregivers to stimulate reflection and discussion, and to benefit patient care.
\end{abstract}

\section{KEYWORDS}

Silence; spiritual care; palliative care; end of life; caregiver

\section{Introduction}

At the end of life silence often takes an increasing prominence in caregiving encounters. For a dying person this may be due to disease progression, medical intervention, a process of withdrawal from the world, grief or shock. For them, their family members and caregivers, the situation sometimes seems to be beyond the capacity of words. It is a natural place for silence but lack of familiarity with the phenomenon may lead to anxiety and avoidance. Greater understanding of the contribution of silence to care has the potential to support professional caregiving practice. 
This paper explores the contribution of silence to spiritual caregiving in palliative settings drawing on research that I (Lynn Bassett) undertook in the UK between 2013 and 2016. The study arose out of my desire, as a palliative care chaplain, to better understand the silences that occur during spiritual caregiving encounters with patients and their family members, silences which seem to be helpful to them and to the caregiving relationship. This personal interest was galvanized by a realization that opportunities for silence may be diminishing in a Western culture that is increasingly focused on activity, communication and information. Aldous Huxley wrote of the twentieth century: 'all the resources of our miraculous technology have been thrown into the current assault against silence' (Lane 2006, 29). Hughes $(1985,97)$, a Jesuit priest and spiritual writer, offers an explanation: 'We are so afraid of silence that we chase ourselves from one event to the next in order not to have to spend a moment with ourselves, in order not to have to look at ourselves in the mirror.'

This trend has been noted in healthcare too. Swift $(2014,167)$, a healthcare chaplain, writes: 'In a world full of productive knowledge and solution focused health care it can be hard to argue for the necessity of space and silence'. Yet the value of silence and space is recognized in religious and spiritual traditions, and in a growing body of evidence for meditative practices, such as mindfulness (Kabat-Zinn 2005), for relief from physical pain and for psychological wellbeing.

There seems to be little evidence for silence as an element of care. Existing knowledge of silence in this context appears to reside mainly in the 'tacit' domain; tacit knowledge is described by Polanyi (1966) as intuitive understanding that is less easily put into words. One objective of this research was to explore and explicate tacit knowledge in the lived experience of palliative care chaplains.

\section{Background}

Spirituality eludes a single, agreed definition (Nolan and Holloway 2014). In healthcare contexts, it is described as the dimension of life that seeks meaning, purpose and transcendence (Puchalski and Romer 2005; Nolan 2012) and is also bound up in the relationship with self, others and with creation. Spiritual care has been defined as: 'That care which recognises and responds to the needs of the human spirit when faced with trauma, illhealth or sadness' (NHS Scotland 2009, 1).

Both spiritual care and palliative care prioritize holistic, person-centred care and emphasize a value in 'being with' another person. Such value transcends the taskrelated focus of 'doing something' for them (Edwards et al. 2010; Tornøe et al. 2014). Spiritual care is recognized as part of the remit of all members of the palliative care team at the level of individual ability (Marie Curie Cancer Care 2003) but is acknowledged as the specialism of chaplains (AHPCC 2013). For this reason, and for homogeneity, this research focused on the lived experience of palliative care chaplains.

Silence is sought and respected in many world faiths and spiritual traditions (Lane 2006). In Christian spirituality it is associated with contemplative and monastic practices. In Catholic seminaries, cultivation of interior silence is described as central to spiritual formation (Keating 2012). Similarly, Clayton (2013), a hospice chaplain, found that a prior condition of interior space is a prerequisite to care. More widely, in nursing and social care, it has been suggested that a regular meditative spiritual practice for caregiving 
staff benefits themselves and the patients they care for (Wright 2006; Wong 2013); but the contribution of silence to the practice of spiritual care has been less well explored.

\section{Literature review}

The review sought empirical research and other published material reporting experience of caregiving silence in interactions between professional caregivers and their patients or clients in clinical or pastoral settings. A systematic search strategy designed for PsycINFO was adapted for the following cross-disciplinary databases: Academic Search Complete, AMED, CINAHL, Medline, Index to Theses, International Bibliography of the Social Sciences and ProQuest Digital Dissertations. This was supplemented by hand-searching, in discipline specific journals including the Journal for the Study of Spirituality, and citation tracking. Eighteen papers were retained for synthesis. Three report empirical research into the experience of psychotherapists in America and the UK (Hill, Thompson, and Ladany 2003; Ladany et al. 2004; Barber 2009). One (Tornøe et al. 2014) explores Norwegian hospice nurses' experience of consoling presence but silence emerged in the findings, not as the subject of the study. The remaining 14 articles provide data in the form of reflection on first-hand experience of silence in caregiving settings including nursing, pastoral care, counselling and psychotherapy. Two articles of particular note are from palliative care in America: Back et al. (2009) describe a 'compassionate silence' derived from contemplative practice and Capretto (2015), a hospice chaplain working with bereavement, theorizes that silence acknowledges the limits of empathetic language in grief and loss.

To synthesize findings from these disparate sources a meta-ethnographic lines-of-argument approach was used, informed by Noblit and Hare (1988). This two-step process compares themes and concepts across studies, clustering similar findings and noting differences. It then draws together the main arguments of each paper to develop an inference of the meaning of the whole, in this case silence as an element of care, based on selective studies of the parts (Noblit and Hare 1988, 62). Silence is acknowledged as a complex and multi-faceted phenomenon (Hill, Thompson, and Ladany 2003; Ladany et al. 2004) that can lead to therapeutically rich moments (King 1995; Himelstein, Jackson, and Pegram 2003). Positive experiences of silence are described as comfortable, affirming and safe (Hill 2004; Back et al. 2009) but silence can also be anxiety provoking, difficult or uncomfortable (Sabbadini [1991] 2004).

Three areas of focus emerged: the relationship between silence and speech, the use of silence and the practice of silence. When understood and used skilfully, silence presents not as an absence of speech (Bravesmith 2012; Capretto 2015) but as an active presence, a 'container of words' (Sabbadini [1991] 2004, 229) or the ground from which speech arises and connections are made (Denham-Vaughan and Edmond 2010). Authors also note that silences may be avoided, especially in Western culture where speech is prioritized (Harris 2004; Moriichi 2009) and that effective use of silence demands experience, training and practice (Hill, Thompson, and Ladany 2003). In psychotherapy, many reasons for the use of silence are identified (Ladany et al. 2004) but Hill, Thompson, and Ladany (2003) conclude that specific recommendations cannot be made about when to use silence; this is a decision based on timing and client needs. Beyond use, Barber (2009) suggests that silence is an experience to be entered into by both caregiver and client. Silent presence has a quality of being fully in the here-and-now, being present to embodied self 
(Denham-Vaughan and Edmond 2010) and being there for the other person in the encounter. In palliative care, it is described as a deeply personal and relational practice (Tornøe et al. 2014) and is observed to foster healing (Back et al. 2009). Being, and staying, in silence with another person at a difficult time for them is described as an act of non-abandonment (Moriichi 2009; Capretto 2015), demonstrating a willingness to remain, even in an uncomfortable place (Himelstein, Jackson, and Pegram 2003; Back et al. 2009).

The resulting interpretation found caregiving silence to be an aid to therapeutic communication, working in partnership with speech and non-verbal modes to enable deep communication beyond the limits of language. A distinction was noted between the 'use of silence' and 'a practice of silence' described by Back et al. $(2009,1113)$ as 'the quality of mind the clinician contributes to the encounter'. This type of silence lends itself, particularly, to the spiritual and existential dimensions of communication where words may fail. The interpretation suggests that silence may be helpful in end-of-life care where speech can be compromised and spiritual care is integral to holistic palliative care. No research into silence in spiritual care at the end of life has been found. Thus, the review highlighted a gap in knowledge that could be addressed by empirical exploration into the specialist lived experience of palliative care chaplains.

\section{Research question, aims and objectives}

The research question was: 'What is the nature, meaning and value of silence in spiritual caregiving at the end of life as experienced by palliative care chaplains?' The aim of the research was to deepen understanding of silence as an element of palliative spiritual care. The objectives were to explore and explicate chaplains' lived experience; to describe and interpret essential qualities of a type of silence that contributes to spiritual care; and to stimulate reflection and dialogue about the value of silence in palliative spiritual care.

\section{Methodology and method}

This study was undertaken in two phases in order to explore, first, my own lived experience of silence (i.e. Lynn Bassett) and, second, the experience of other palliative care chaplains. The research paradigm was grounded in a contemplative construction of reality proposed by Braman (2007) in communication theory. A contemplative construction encompasses dimensions of reality beyond the boundaries of the social world; inwardly it begins in embodied self and outwardly it looks to the transcendent. Thus it lends itself to the phenomenon of silence which is both 'embodied by' and 'external to' human beings.

A Heideggerian understanding of phenomenology was adopted. This capitalizes upon the subjective relationship between researchers and their research projects. It acknowledges that researchers bring their own history of interpreted experience and engage actively in the process of deepening understanding. This demands reflexivity (Etherington 2004) in order to establish and maintain an awareness of personal biases and assumptions that can limit the possibilities for discovery. Gadamer (1976) describes these limitations as a personal horizon; when acknowledged reflexively, it is possible for other horizons to come into view and new and different dimension of meaning to be uncovered (Van 
Manen 1990). Gadamer theorizes that it is from the fusion of multiple horizons that an interpretation may emerge which strives for truth.

The strong emphasis on researcher involvement informed the decision to design the research in two phases. A reflective journal entry explains:

As both palliative care chaplain and researcher, I needed to explore my own understanding of silence in my spiritual life and in my professional world of palliative spiritual care. Only in the light of this expanded self-awareness, with the recognition of my own pre-assumptions and personal horizon (Gadamer 1976), would I be open to the new dimensions of meaning that may arise from the experience of other palliative care chaplains (Bassett: Journal 16/01/2013)

The purpose of Phase One was to establish and explicate my own horizon of understanding of silence as an element of palliative spiritual care, prior to embarking on the process of describing and interpreting essential qualities of this type of silence, from the lived experience of other chaplains in Phase Two. Heuristic inquiry, a method of self-inquiry proposed by Moustakas (1990), was used to explore and explicate my own experience of silence from both a personal and professional perspective. It began with a three-day silent retreat to immerse myself in a silence familiar to my own spirituality. The process continued with in-depth reflection on my professional experience of silence in 11 spiritual care encounters with patients and family members recorded in a reflective journal.

Having arrived at an interpretation of my own understanding, experience was sought from three other palliative care chaplains, for comparison with my own and to expand the horizon of understanding. Moustakas (1990) suggests that, in the cooperative sharing of a heuristic inquiry interview, both parties may open pathways to the other for deeper understanding of the phenomenon being explored. Interviews were unstructured; this allowed flexibility for the conversations to take their own course. A topic guide was used to provide prompts, where needed. It included definitional questions such as: 'Is silence a part of your own spirituality (or has it ever been)? Can you tell me more ...' and in the core discussion 'Can you think of a time when you experienced silence with a patient or family member?' A manual process of engagement with the data, as outlined by Moustakas (1990), included periods of immersion and incubation which led to illumination and explication. Individual depictions of each participant's experience were developed and agreed with participants before the composite depiction - which highlights universal qualities and themes of silence as an element of end-of-life spiritual care - was composed.

Participants for both phases were recruited through the Association of Hospice and Palliative Care Chaplains (AHPCC). A purposive sampling approach aimed to recruit a cohesive cohort of information rich participants (Pascal 2010). An invitation email, with a participant information pack attached, was sent to regional representatives of four AHPCC regions with a request to circulate to members. Criteria for inclusion were that the participant should be currently in post as a palliative care chaplain and have at least one year's experience in end-of-life spiritual caregiving. A secondary aim was to strive for an overall balance between male and female. All respondents who met recruitment criteria were interviewed, resulting in a total of 15 participants (nine male and six female) across the two phases. The sample size takes into account the in-depth engagement with the data demanded in a phenomenological study. Interviews were digitally recorded and transcribed by the researcher to gain early familiarity with the data. They ranged between 41 and $89 \mathrm{~min}$ in duration; Moustakas (1990) particularly emphasizes 
the importance of giving participants the opportunity to tell their story to a point of natural closing.

In Phase Two, specific examples of silence in spiritual caregiving encounters with patients and their family members were sought from 12 more palliative care chaplains. Hermeneutic phenomenology, following Van Manen (1990), was used to describe and interpret essential qualities of silence as an element of end-of-life spiritual care. Van Manen $(1990,107)$ describes essential qualities as 'those which make a phenomenon what it is and without which the phenomenon could not be what it is'. There is no prescribed method for data analysis in hermeneutic phenomenological research (Finlay 2009) but Van Manen (1990, 93) suggests methods to uncover thematic aspects of a phenomenon. In this research two of these approaches were adopted: 'wholistic reading', where attention is given to the participant's account as a whole; and 'selective reading' where statements that seem to be particularly revealing about the phenomenon are highlighted and extracted. In this manual process of analysis, emergent themes were identified and clustered to reveal overarching themes that described the essential qualities of silence as an element of end-of-life care.

\section{Ethics and quality}

Permission to conduct the research was granted by Lancaster University Ethics Committee in 2013. Measures taken to protect the wellbeing of participants, the researcher and other third parties included provision of information to participants; obtaining participant consent; anonymization of identifiers; protection of confidentiality; and secure data storage.

In a descriptive and interpretative methodology, such as phenomenology, trustworthiness is considered a marker of quality (Polkinghorne 1983); this is achieved through a clear, cohesive and transparent approach. Both Moustakas (1990) and Van Manen (1990) emphasize the commitment, discipline and rigour involved in the research process which is located largely in the person of the researcher (Moustakas 1990). Phenomenology does not seek to provide definitive answers; the aim is to produce rich description which draws the reader into the researcher's discoveries, moving them to recognize the phenomenon from their own experience (Van Manen 1990). This relies upon in-depth reflection on the data, and use of verbatim material. Knowledge is not presented as fact but is understood to be 'contingent, proportional, emergent and subject to alternative interpretations' (Finlay 2009, 17). In this research, Phase One participants checked and agreed the depictions of their experience to assure accuracy and transparency and co-authors were engaged at all stages to ensure consistency, challenge bias and maximize the potential of the reflective process.

\section{Findings}

Findings from Phase One of the research contributed to a horizon of understanding of silence explicated from palliative care chaplains' personal experience and spiritual caregiving practice. The process of self-inquiry revealed that my own personal and professional experiences were linked by a common understanding of silence as an interior space: a place of giving of self, time and attention to another person in a spiritual caregiving relationship or to God in personal prayer. Themes of silence that emerged are illustrated in Figure 1: they are stillness, listening, connection, witness, companionship and 
communication. These are underpinned by a notion of depth. Increasing depth of silence seems to afford increasing intensity of experience and potential for self-discovery. For me, silence emerged as both a rich and challenging phenomenon. Reflective journal accounts describe the experience of being with people who had been silenced by their own illness or grief; noticeable in these accounts are my own feelings of helplessness and vulnerability summarized as:

... not knowing what to say, feeling that I have nothing to offer, an uncomfortableness in notdoing and, in shared silences, the beginnings of something that might be recognised as compassion, from the Latin com-passio, literally the experience of suffering with another person.

(Bassett 2016, 97)

The experience of three other palliative care chaplains helped to expand the horizon of understanding by adding four pairs of divergent themes: silence is both interior and external; comfortable and uncomfortable; it can foster a sense of connection and be used to communicate rejection; it can provide a caregiving space and be used as a caregiving tool or intervention (see Figure 1).

The participating chaplains, Susan, Paul and John (all names are pseudonyms), confirmed that silence is important to them personally and as a resource for their professional practice. Their experience of the value of silent time and space seemed to motivate them to make opportunities for other people to find a time of stillness for themselves. Susan used the term 'engineer' to describe her attempts to introduce silence into the caregiving relationship, assisted by touch, body language or holding a hand. Paul confirmed, 'silence is an invaluable part of what we offer with people and if it weren't there a lot would be missing'. They identified silence as a space which allows things to happen. John explained:

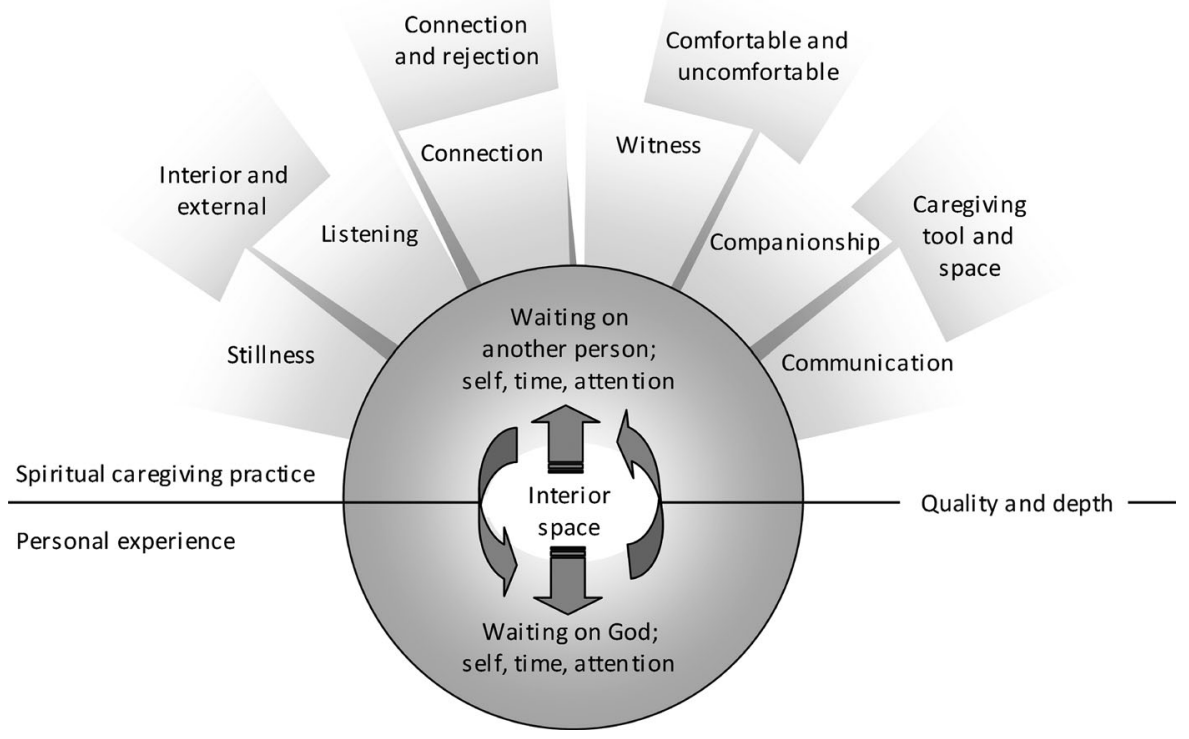

Figure 1. Horizon of palliative care chaplains' understanding of silence in personal experience and spiritual caregiving practice. 
I'm holding the silence for them, because I'm assuming that there is something going on for them. And then it becomes a very intimate sharing of silence and [afterwards] we may or may not talk about what's happened in the silence but there's, hopefully, a mutual recognition that there's something going on.

Silence seems to foster a sense of connection between the two people in the caregiving relationship. Participants observed that it also offers a space for connection with God. Susan described the tangible nature of this connection, saying, 'you can almost see it, feel it ... in the silence'.

The experiences related by the other chaplains supported much of the understanding gained from self-inquiry: themes of connection, stillness and listening were common and enriched the data. Participants used analogous words to describe their experience of silence: stillness, space, calm and quiet but they also noted differences in quality. For example Susan distinguished 'quiet' from 'quality silence'; this resonated with my discovery that silence has a dimension of depth. The horizon of understanding, at the end of Phase One, is illustrated in Figure 1.

Phase Two of the research, which explored palliative care chaplains' lived experience of silence in end-of-life caregiving encounters, resulted in three overarching themes: silence as a way of being with another person; silence as a medium for communication; and silence as an enabler of change. In each theme, increasing depth of silence was linked with an increasing intensity in the inter-personal relationship and in the content of the spiritual caregiving encounter. This is illustrated in Figure 2 and described, with examples, below.

\section{Silence as a way of being with another person}

This primary theme begins with a disposition of self in relationship with the other person in an encounter. Participants described a type of silence that feels comfortable and companionable. The term 'just sitting' with another person occurs in all but two of the transcripts; it is described as a way of being, which is not only silent but also includes a physical

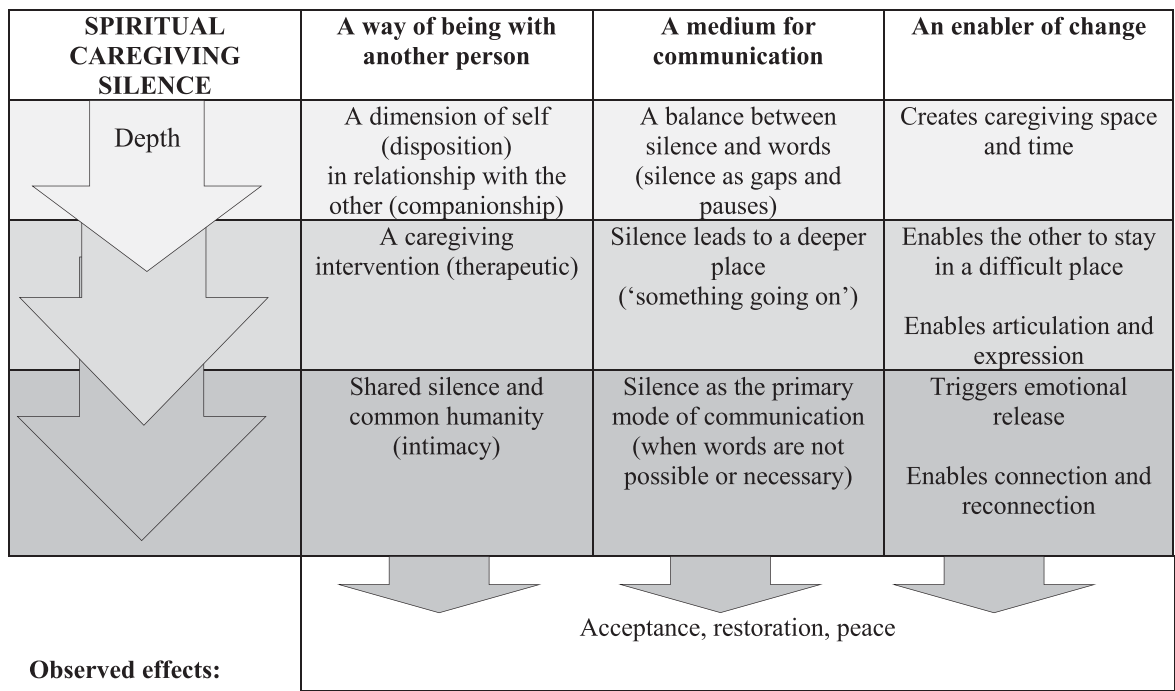

Figure 2. Themes of spiritual caregiving silence demonstrating increasing depth of experience. 
quality of stillness, that does not interrupt but offers companionable presence. Reflecting on her own experience of illness, Julia recalled, 'you don't always want to talk about how you're feeling or your illness. But equally, you'll love to have a presence with you'. As a dimension of self, silence can also be used as a caregiving intervention. This is illustrated by Jonathan who described his use of embodied silence as a therapeutic tool: 'it's about being silent myself in order to bring a comfortable feeling into the room'. As shared silence deepens, some participants used the term 'intimacy' to describe the sense of common humanity that they experienced. Alison recalled one such 'contemplative encounter' with a patient: 'It was like that feeling when two people are in love and there's the whole world going on but ... you're in your own little world'. Participants suggested that when two individuals share silence, they enter into a mutual space in which both have the potential to be changed. Tom alluded to humanistic psychologist Carl Rogers when he explained:

It's about your client having a desire for change, and me willing to be part of that change. It needs two people to work together. It's not just that I am giving to somebody. They are also giving to me. It's not a one way flow.

\section{Silence as a medium for communication}

In all accounts the closely inter-woven relationship of silence with speech and non-verbal signs is clearly illustrated. Several participants noted that, even after a person has died, professional caregivers often introduce themselves to the patient verbally before sharing in their silence. In earlier and more general conversations, speech tends to take priority and silence is present as pauses and gaps between phrases. Participant Chris described these as 'pockets of silence' that help to build trust and rapport. He explained how a change in the quality of silence can mark a gear-change in the conversation using, as an example, a visit to an elderly patient, Marjorie, who seemed to have something on her mind. As the conversation progressed the silences between sentences lengthened. When Marjorie began to engage with her deepest feelings Chris experienced a sudden silence. He recalled that came as if with a 'thud'. The impact made him feel quite uncomfortable but he recognized the importance of holding that silence for her so that she could stay with her thoughts and feelings for as long as she needed. The recognition of 'something going on' for the other person in the spiritual caregiving encounter was highlighted in a number of accounts.

Other participants recalled situations where patients physically could not speak; then silence became the primary mode of communication. Clare described sitting in silence with Michael, who had a neurological condition, during regular visits over an eight month period. She explained:

He was able to receive gaze ... So we were able to sit and look into each other's eyes and hold hands in a way that you often only do with lovers. But we did that in absolute silence and respectfulness. And it was as if Michael understood that I could manage the emotion of his illness ... that I could see his suffering.

\section{Silence as an enabler of change}

There was a common understanding in all the accounts that silent presence can create caregiving space and time. Several participants noted that chaplains are seen to embody 
silence, not simply as a state of mind but as a whole way of being. Tom introduced the concept of 'unspokenness', a silencing of self and an economy of words, using only sufficient language to ensure that the other person feels safe and accepted. This may present a counterpoint to the atmosphere of anxiety that sometimes accompanies end-of-life situations. James noted that an intentional silence also resists the natural desire to say something to 'take away their fears, or anxieties or worries or whatever'. His suggestion that well-meaning words can also take away something from the person her or himself was echoed in other participant's accounts. Chris reflected on the close care of Marjorie's family: 'It seemed like they were attaching themselves to her, like a hug around the bed, but one which actually stopped her from saying what she wanted to say'.

Demonstrating the value of silent caregiving space, Charles described a meeting with Bill who was trying to come to terms with the shock of his terminal diagnosis. Charles was conscious of the importance of allowing Bill the time that he needed. He explained: The silences were allowing me to give him the space to speak about what mattered. The silences were being able to live with the silences that happen in life when there are no answers.

Other participants described how their silent presence enabled relatives to stay with their loved one to the end. They noted that, around the time of death especially, words seem to be redundant and that silence allows a professional caregiver to be alongside as companion and witness without being an intrusion. Describing one particular occasion, Simon explained his silence as recognition of, and reverence for, a deeply personal moment for a dying woman and her husband:

There was just silence in the room. You couldn't even hear the patient breathing. It was just peaceful. I think I'd been in the room half an hour. And I'd said my couple of prayers in the first five or ten minutes. The rest of the time was just being there ... I said nothing because I literally couldn't think of a thing to say. And not only that, [in a whisper] I thought it was not my place to say anything.

Other accounts contained examples of patients who, in accompanied silence, found the courage to articulate the reality of their own dying. In this acknowledgment they were enabled to come to greater acceptance of their situation and continue with their remaining life. Amanda shared the story of her visit to a dying woman who had been referred because of her deep denial. Amanda explained how, by carefully listening to her story and then holding a long silence, which began to feel quite uncomfortable for her, the patient finally acknowledged the truth: 'I think I'm dying', and a significant conversation followed. This enabled her to talk with her family and Amanda concluded, 'she sorted out lots of things ... and she died really peacefully'.

Where speech is not possible a silent accompanied space can allow non-verbal expression. Participant Jill shared the story of Nigel whose rapid degenerative illness left him with no speech and no movement; she described how times of silence with her in the chapel gave him a 'buffer' to cope. He communicated his anger and frustration through his eyes and Jill sensed that he gained some relief from her non-verbal confirmation that he had been 'heard'.

Many accounts describe tears; caregiving silence has the potential to trigger emotional release. Jonathan explained, 'It is the quality of pausing. What you're offering is space, silence offers space in time. You're pushing the pause button and that releases emotions'. 
Several participants were visibly moved as they recalled these encounters, reflecting the depth of connection forged in silence.

As a dimension of spiritual care, participants identified silence as sacred space. Transcending connection on a human level, in silent moments with patients and family members, they witnessed connection or reconnection with God. Simon presented a Christian theological position that chaplains bring the presence of God to the encounter. Amanda described the need for 'opening up the deepest part of ourselves [as caregivers] for God's presence'. Reflecting Phase One findings, a number of accounts refer to a silence that follows spoken prayers. Steve recounted a simple ritual with a patient who asked for affirmation and prayers at the end of her life. His own experience was that silence released a presence of love into the room ... a real sense of common union and a tangible presence of the immanence of mystery and love' and concluded 'there was nothing more to say'.

\section{Observed effects}

Participants described beneficial changes in the people with whom they have shared silent encounters. Jonathan reported ' $M y$ silence helped them to be calm; I've heard that from families'. Others noted that the opportunity to share thoughts and feelings led patients and family members to acceptance; afterwards they appeared to be more at peace. Participant Steve concluded: 'To find a stillness allows for restoration, or even a new comprehension, within the person, of their worth and value simply as a human being'. 'Spiritual caregiving silence' emerged as a way of being with another person that evokes a sense of connection and companionship and provides a means of, and medium for, communication beyond the capacity of the spoken word. Further, a silent, caregiving space has the potential to enable change; this may begin with the expression or articulation of a deep personal truth. Participants observed that this process helps the recipient of spiritual care to move forward towards acceptance, restoration and peace.

\section{Discussion}

The findings contribute in-depth understanding of the nature, meaning and value of silence in spiritual caregiving at the end of life. The interpretation is further illuminated by experience in other related disciplines, as discussed below, but is particular to the practice of spiritual care. It was confirming to discover that spiritual caregiving silence reflects qualities of spiritual care itself. Consistent with the purpose of spiritual care, which is described in the background to this study as person-centred with an emphasis on being with another person (Edwards et al. 2010), the research found the nature of spiritual caregiving silence to be a way of being with another person. As a way of being, silence has different modes that involve the caregiver as both actor, by being silent, and participant by being in or with the silence of another person. This interpretation is informed by the relationship between interior and external silence identified by Keating (2012), in his article on interior silence as part of seminary formation, and further illuminated by the work of Davies $(2008,201)$ who, from the perspective of negative theology, highlights the limitations of the English language to express these different dimensions of silence. He turns to the Russian words tishina, translated as 'the silence of the forest' to denote stillness or absence of sound and molechanie as the silence of one who ceases speaking. Davies 
$(2008,201)$ suggests that 'the two silences are in constant tension and their relation can be a richly ambiguous one'.

In this research the meaning of silence, as a way of being with another person, is derived from the sense of connection and companionship that is evoked. In the particular setting of palliative care, the data provide examples of the value of caregiver silence as companionable but non-intrusive presence. This is especially relevant when patients are physically unable to speak or when they, and their family members, are rendered speechless by their situation. It resembles the state of active passivity noted by Lunn (2009) in spiritual direction, which she describes as not to be mistaken for doing nothing but an intentional choice to be still, to wait and to attend. Lunn $(2009,225)$ writes of attending as an act of kenosis (self-emptying); she suggests this is 'a deeply theological act (...) It involves putting aside one's own thoughts, feelings and desires in order to attend to the other'.

The research has identified further value in the potential for change that emerges out of silence as accompanied, caregiving space. It manifests as the expression or articulation of deep concerns and, then, the possibility of acceptance and greater peace. This reflects and transcends the understanding of the phenomenon in psychoanalytic literature: Sabbadini ([1991] 2004, 229) describes silence as 'a container of words' but, in this research, silence has been observed to enable expression of meaning without the use of words; for example in Jill's encounters with Nigel whose condition denied him the luxury of speech. The understanding of the value of silence as caregiving space is resonant with the typology of 'compassionate silence' described by Back et al. (2009). This research has found that silence is not only a state of mind but is 'embodied' as a therapeutic tool. To be in silence, with the silence of another person, is to participate in a shared silence. Participants' experience is that, when the other person is suffering, this can be an uncomfortable place but, consistent with existing literature (Himelstein, Jackson, and Pegram 2003; Back et al. 2009; Capretto 2015), they recognize the value in staying in the silence with the other person.

This research has found that being in shared silence together enables the other person in the caregiving relationship to be with her or himself in a way that may not be possible when alone. It builds upon the findings of Capretto $(2015,353)$, a chaplain working with bereavement, that 'silence provides a transitional medium for the spiritual and intrapsychic process of the loss, which cannot be effected in isolation'. In this research the theory is supported by accounts of relatives who were enabled to stay with their dying loved ones till the end, and examples of chaplains' silent presence that enabled patients close to the end of life to reflect on and articulate their own deepest concerns. Hughes (1985) infers that people are afraid of silence because of the opportunity it offers to engage with self, yet chaplains in this study observed that it is the opportunity for engagement with self, in a safe accompanied space, that enables the other in the relationship to express deep truths and this can lead to a greater sense of acceptance and peace. Thus, this research supports the conclusion of the psychoanalyst Sabbadini ([1991] 2004, 239) 'Perhaps, staying with our patients' and our own silences a little longer is the one unambiguous recommendation I can honestly make'.

\section{Conclusion}

This research has explored and expanded the understanding of silence as an element of palliative spiritual care. It draws together specialist expertise and cross-disciplinary experience 
incorporating understandings of silence from spirituality, communications theory, and psychotherapy and counselling to illuminate the nature, meaning and value of spiritual caregiving silence within the holistic practice of interdisciplinary palliative care. Spiritual caregiving silence includes types of silence used and practiced in wider palliative caregiving and in other disciplines but, like spiritual care itself, it is distinctive in purpose and practice.

The phenomenological methodology supports the explication of the lived experience of chaplains in a reflective and congruent way. It is recognized that the specialist role of palliative care chaplains may influence the context and character of the caregiving encounter. A limitation of the research may be that it does not include the experience of other professional caregivers who also have a responsibility to offer spiritual care. This could be usefully explored in future research, as could the experience of recipients of spiritual care. One purpose of hermeneutic phenomenology is to seek essential qualities of a phenomenon. Spiritual caregiving silence is what it is because it occurs in a spiritual caregiving context and, as findings from this research indicate, spiritual caregiving silence has the potential to enhance the quality of spiritual care.

This study offers a variety of examples of the contribution of silence to spiritual caregiving at the end of life: as a way of being with another person, silence can offer comfortable companionship; as silence deepens a sense of intimacy and connection may be evoked; and, when words fail, silence can provide a means of and a medium for communication. In addition, silence creates a caregiving space where the other person in the spiritual care relationship may be enabled to be with him or herself in a way that he or she may find helpful and healing. For the caregiver, silence is a demanding skill and practice. Nevertheless, chaplains in this research confirm that its challenges are outweighed by the spiritual caregiving intention to stay with another person and the perceived value to patient care. Whilst offering a unique insight into the experience of chaplains and the particular setting of end-of-life spiritual care, these findings may resonate with other spiritual caregiving situations and the experience of other caregivers to stimulate reflection and discussion and further deepen understanding to benefit patient care.

\section{Disclosure statement}

No potential conflict of interest was reported by the authors.

\section{Notes on contributors}

Lynn Bassett is a retired healthcare chaplain from the south east of England. Her interest is in the phenomenon of silence as part of human spirituality and, particularly, in the context of palliative spiritual care.

Amanda F. Bingley is Lecturer in Health Research in the Division of Health Research, Faculty of Health \& Medicine at Lancaster University, UK.

Sarah G. Brearley SFHEA is Senior Lecturer in Health Research at the International Observatory on End of life Care, Lancaster University, UK.

\section{References}

AHPCC (Association of Hospice and Palliative Care Chaplains). 2013. Guidelines for Hospice and Palliative Care Chaplaincy.3rd ed. [pdf]. www.ukbhc.org.uk/ ... /ahpcc_standards_for_hospice_ and_palliative_care_chaplaincy. 
Back, A. L., S. M. Bauer-Wu, C. H. Rushton, and J. Halifax. 2009. "Compassionate Silence in the Patient-clinician Encounter: A Contemplative Approach.” Journal of Palliative Medicine 12 (12): 1113-1117.

Barber, T. 2009. "Newly Qualified Counsellors' Experience of Silence within the Therapeutic Setting." Unpublished Master's thesis, Roehampton University, UK. http://www.academia.edu/ 362080/Silence_in_Psychotherapy_Newly_qualified_counsellors_experience_of_silence_ within_the_therapeutic_setting.

Bassett, L. 2016. "Spiritual Caregiving Silence: An Exploration of the Phenomenon and Its Value in End-of-life care.” PhD thesis., Lancaster University.

Braman, S. 2007. "When Nightingales Break the Law: Silence and the Construction of Reality." Ethics and Information Technology 9 (4): 281-295.

Bravesmith, A. 2012. "Silence Lends Integrity to Speech: Transcending the Opposites of Speech and Silence in the Analytic Dialogue." British Journal of Psychotherapy 28 (1): 21-34.

Capretto, P. 2015. "Empathy and Silence in Pastoral Care for Traumatic Grief and Loss." Journal of Religion and Health 54 (1): 339-357.

Clayton, M. 2013. "Contemplative Chaplaincy? A View from a Children's Hospice." Practical Theology 6 (1): 35-50.

Davies, O. 2008. "Soundings: Towards a Theological Poetics of Silence." In Silence and the Word: Negative Theology and Incarnation, edited by O. Davies, and D. Turner, 201-222. Cambridge: Cambridge University Press.

Denham-Vaughan, J., and V. Edmond. 2010. “The Value of Silence.” Gestalt Journal of Australia and New Zealand 6 (2): 5-19.

Edwards, A., N. Pang, V. Shiu, and C. Chan. 2010. "The Understanding of Spirituality and the Potential Role of Spiritual Care in End-of-life and Palliative Care: A Meta-study of Qualitative Research." Palliative Medicine 24 (8): 753-770.

Etherington, K. 2004. Becoming a Reflexive Researcher: Using Our Selves in Research. London: Jessica Kingsley.

Finlay, L. 2009. “Debating Phenomenological Research Methods.” Phenomenology \& Practice 3 (1): 6-25.

Gadamer, H. G. 1976. Philosophical Hermeneutics. Berkeley: University of California Press.

Harris, A. 2004. “The Experience of Silence: A Client Case Study." Counselling Psychology ReviewBritish Psychology Society 19 (1): 5-12.

Hill, M. 2004. "The Silence of the Body." Journal of Religion and Health 43 (1): 29-43.

Hill, C. E., B. J. Thompson, and N. Ladany. 2003. "Therapist Use of Silence in Therapy: A Survey.” Journal of Clinical Psychology 59 (4): 513-524.

Himelstein, B. P., N. L. Jackson, and L. Pegram. 2003. “The Power of Silence.” Journal of Clinical Oncology 21 (9s): 41s-41.

Hughes, G. W. 1985. God of Surprises. London: Darton, Longman \& Todd.

Kabat-Zinn, J. 2005. Coming to Our Senses: Healing Ourselves and the World Through Mindfulness. London: Piatkus.

Keating, J. 2012. “Seminary Formation and Interior Silence." Nova et Vetera English Edition 10 (2): 307-319.

King, K. C. 1995. "Using Therapeutic Silence in Home Healthcare Nursing." Home Healthcare Nurse: The Journal for the Home Care and Hospice Professional 13 (1): 65-68.

Ladany, N., C. E. Hill, B. J. Thompson, and K. M. O’Brien. 2004. “Therapist Perspectives on Using Silence in Therapy: A Qualitative Study." Counselling and Psychotherapy Research 4 (1): 80-89.

Lane, J. 2006. The Spirit of Silence: Making Space for Creativity. Totnes: Green Books.

Lunn, J. 2009. "Paying Attention: The Task of Attending in Spiritual Direction and Practical Theology." Practical Theology 2 (2): 219-229.

Marie Curie Cancer Care. 2003. Spiritual and Religious Care Competencies for Specialist Palliative Care [pdf]. http://www.mariecurie.org.uk/Documents/HEALTHCARE-PROFESSIONALS/ spritual-religious-care-competencies.pdf.

Moriichi, S. 2009. "Re-discovery of Silence in Pastoral Care." The Journal of Pastoral Care and Counselling 63 (1-2): 1-6. 
Moustakas, C. 1990. Heuristic Research: Design, Methodology and Applications. Newbury Park, CA: Sage.

NHS Scotland. 2009. Spiritual Care and Chaplaincy. Edinburgh: The Scottish Government.

Noblit, G. W., and R. D. Hare. 1988. Meta-ethnography: Synthesising Qualitative Studies. Newbury Park, CA: Sage.

Nolan, S. 2012. Spiritual Care at the End of Life: The Chaplain as Hopeful Presence. London: Jessica Kingsley.

Nolan, S., and M. Holloway. 2014. A-z of Spirituality. Basingstoke: Palgrave Macmillan.

Pascal, J. 2010. "Phenomenology as a Research Method for Social Work Contexts: Understanding Lived Experience of Cancer Survival." Currents 9 (2): 9-23.

Polanyi, M. 1966. The Tacit Dimension. Chicago: The University of Chicago Press.

Polkinghorne, D. 1983. Methodology for the Human Sciences: Systems of Inquiry. Albany: State University of New York Press.

Puchalski, C., and A. L. Romer. 2005. “Taking a Spiritual History Allows Clinicians to Understand Patients More Fully.” Journal of Palliative Medicine 3 (1): 129-137.

Sabbadini, A. [1991] 2004. "Listening to Silence." British Journal of Psychotherapy 21 (2): 219-240.

Swift, C. 2014. Hospital Chaplaincy in the Twenty-first Century: The Crisis of Spiritual Care on the NHS. Farnham: Ashgate.

Tornøe, K. A., L. J. Danbolt, K. Kvigne, and V. Sørlie. 2014. “The Power of Consoling Presence Hospice Nurses' Lived Experience with Spiritual and Existential Care for the Dying." BMC Nursing 13 (25): 1-8.

Van Manen, M. 1990. Researching Lived Experience: Human Science for an Action Sensitive Pedagogy. London, ON: State University of New York Press.

Wong, Y. L. R. 2013. "Returning to Silence, Connecting to Wholeness: Contemplative Pedagogy for Critical Social Work Education.” Journal of Religion and Spirituality in Social Work: Social Thought 32 (3): 269-285.

Wright, S. 2006. “The Beauty of Silence.” Nursing Standard 20 (50): 18-20. 